\title{
Author Correction: Quantum guidelines for solid-state spin defects
}

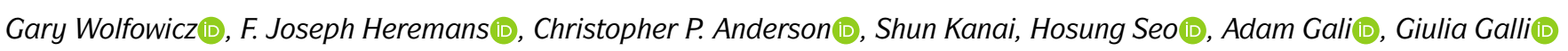
and David D. Awschalom (1)

Correction to: Nature Reviews Materials https://doi.org/10.1038/s41578-021-00306-y, published online 26 April 2021.

The caption for Figure 5a was incorrectly published as "Various types of spectral diffusion depending on the noise correlation time: (1) Gaussian broadened line $\left(\tau_{c}<\tau\right),(2)$ discrete spectral jumps and (3) spectral wandering." The corrected text is "Various types of spectral diffusion depending on the noise correlation time: $(1)$ discrete spectral jumps, $(2)$ Gaussian broadened line $\left(\tau_{c}<\tau\right)$ and $(3)$ spectral wandering”. This has been corrected both in the PDF and in the HTML versions of the article.

https://doi.org/10.1038/s41578-021-00393-x I Published online 14 October 2021

(c) Springer Nature Limited 2021 